\title{
UPPER BOUNDS \\ FOR DOUBLE EXPONENTIAL SUMS ALONG A SUBSEQUENCE
}

\author{
Christopher J. White
}

\begin{abstract}
We consider a class of double exponential sums studied in a paper of Sinai and Ulcigrai. They proved a linear bound for these sums along the sequence of denominators in the continued fraction expansion of $\alpha$, provided $\alpha$ is badly-approximable. We provide a proof of a result, which includes a simple proof of their theorem, and which applies for all irrational $\alpha$.
\end{abstract}

Communicated by Michael Drmota

\section{Introduction}

\subsection{Some notation}

Let $\alpha=\left[a_{0} ; a_{1}, \ldots\right]$ denote the continued fraction expansion of $\alpha \in \mathbb{R} \backslash \mathbb{Q}$. We write $\|x\|$ for the distance from $x$ to the nearest integer. The convergents $p_{n} / q_{n}=\left[a_{0} ; a_{1}, \ldots, a_{n}\right]$, where $\left(p_{n}, q_{n}\right)=1$, give good approximations to $\alpha$. We call $\left\{q_{n}\right\}_{n \in \mathbb{N}}$ the sequence of denominators of $\alpha$. We say that an irrational number $\alpha$ is badly-approximable if there exists $\varepsilon_{\alpha}>0$ such that for all $p, q \in \mathbb{Z}$, $(p, q)=1$, we have

$$
\left|\alpha-\frac{p}{q}\right|>\frac{\varepsilon_{\alpha}}{q^{2}} .
$$

These correspond precisely with those numbers $\alpha$ for which there exists $N \in \mathbb{N}$ such that, $a_{n}(\alpha) \leq N$ for all $n \in \mathbb{N}$. The set of all badly-approximable numbers is a set of Lebesgue measure zero.

2010 Mathematics Subject Classification: 11J70, 11L03, 11L07.

Keywords: continued fraction, badly-approximable $\alpha$, double-exponential sum, discrepancy, Koksma-Hlawka inequality, Ostrowski expansion.

The author's research is supported by an EPSRC Doctoral Training Grant. 


\section{CHRISTOPHER J. WHITE}

When $\alpha$ is badly approximable, we have the helpful bound that

$$
\left\|q_{n} \alpha\right\|>\frac{\varepsilon_{\alpha}}{q_{n}} .
$$

Since convergents give the best approximations for the distance to the nearest integer (see [7]), this means that for $m \leq q_{n+1}-1$ we have the bound

$$
\|m \alpha\|>\frac{\varepsilon_{\alpha}}{q_{n}}
$$

We write $f(n)=O(g(n))$ to mean that there exists a constant $C$, (which does not depend on $n)$, such that

$$
f(n) \leq C \cdot g(n) \text { for all } n \in \mathbb{N} .
$$

Finally, we define the discrepancy of a sequence.

Definition 1.1. Let $\left(x_{n}\right)$ be a sequence of real numbers. For $N \in \mathbb{N}$ the discrepancy of $\left(x_{n}\right)$ modulo one, $D_{N}\left(x_{n}\right)$, is defined as:

$$
D_{N}\left(\left\{x_{m}\right\}\right):=\sup _{I \subseteq \mathbb{R} / \mathbb{Z}}\left|\sum_{m=1}^{N} \chi_{I}\left(x_{m}\right)-N \cdot\right| I||,
$$

where $I$ denotes an interval and $\chi_{I}$ is the characteristic function of $I$.

\subsection{Double exponential sums}

In [9] S in a i and Ulcgrai studied double trigonometric sums of the form

$$
T_{M}(\alpha)=\frac{1}{M} \sum_{m=0}^{M-1} \sum_{n=0}^{M-1} e(n m \alpha) .
$$

We want to determine when the absolute value of this sum is bounded uniformly (i.e., by a constant which depends only on $\alpha$ ) over some subsequence

$$
M \in \mathscr{A} \subseteq \mathbb{N} .
$$

This will, obviously, depend on the Diophantine properties of $\alpha$ and the subsequence $\mathscr{A}$.

We will see that the problem of bounding this sum depends importantly on controlling sums such as

$$
\left|\frac{1}{M} \sum_{m=1}^{M} \frac{1}{\{\{m \alpha\}\}}\right|
$$


Here

$$
\{\{x\}\}:= \begin{cases}\{x\}, & x \in\left[0, \frac{1}{2}\right], \\ \{x\}-1, & x \in\left(-\frac{1}{2}, 0\right),\end{cases}
$$

is the signed fractional part of $x \in \mathbb{R}$.

In [9] the following is proved

Theorem 1.2 (Sinai, Ulcigrai). Let $\alpha$ be badly-approximable. Consider the following double trigonometric sum:

$$
T_{M}(\alpha):=\frac{1}{M} \sum_{m=0}^{M-1} \sum_{n=0}^{M-1} e(n m \alpha) .
$$

Then there exists a constant

$$
C=C(\alpha)>0
$$

such that

$$
\left|T_{M}\right| \leq C_{\alpha} \quad \text { for all } \quad M \in\left\{q_{n}\right\}_{n \in \mathbb{N}}
$$

REMARK 1.3. The sum here is an example of a 2-dimensional finite theta sum. In [2] Cosentino and Flaminio prove bounds for far more general $g$ -dimensional finite theta sums. A special case of one of their results implies that the above theorem is true for all $M \in \mathbb{N}$.

Our main theorem generalises Theorem 1.2

TheOREM 1.4. Let $\alpha \in \mathbb{R} \backslash \mathbb{Q}$. Then there exists a constant

$$
C=C(\alpha)>0
$$

such that

$$
\left|T_{q_{n}}\right| \leq C_{\alpha} \cdot \max \left\{\frac{\log \left(2 \cdot \max _{i \leq n}\left\{a_{i}\right\}\right)}{a_{n+1}}, 1\right\} \quad \text { for all } n \in \mathbb{N} .
$$

REMARK 1.5. By examining signs it appears that the upper bound here is close to best possible. Equation (1.13) in 1 gives a lower bound for the largest terms in a sum that we will consider. While it is true that we use the triangle inequality earlier in our calculation, it does not make our estimate so much larger. 


\section{Proof of main result}

\subsection{Reducing $T_{M}$}

Following the methods in [9], we split $T_{M}$ into two separate sums.

By summing the terms for $n=0, \ldots, M-1$, we can rewrite (1.2.1) as

$$
T_{M}=1+\frac{1}{M} \sum_{m=1}^{M-1} \frac{e(M m \alpha)-1}{e(m \alpha)-1} .
$$

Then we can write $T_{M}=1+S_{M}^{\prime}-S_{M}^{\prime \prime}$, where

$$
S_{M}^{\prime}:=\frac{1}{M} \sum_{m=1}^{M-1} \frac{e(M m \alpha)}{e(m \alpha)-1}
$$

and

$$
S_{M}^{\prime \prime}:=\frac{1}{M} \sum_{m=1}^{M-1} \frac{1}{e(m \alpha)-1} .
$$

We will prove that there exist constants $C^{\prime}, C^{\prime \prime} \in \mathbb{R}$ such that

and

$$
\left|S_{q_{n}}^{\prime}\right| \leq C^{\prime} \cdot \max \left\{\frac{\log \left(2 \cdot \max _{i \leq n}\left\{a_{i}\right\}\right)}{a_{n+1}}, 1\right\}
$$

$$
\left|S_{q_{n}}^{\prime \prime}\right| \leq C^{\prime \prime} \text { for all } n \in \mathbb{N} \text {. }
$$

These constants will depend only on $\alpha$.

\subsection{The sum $S_{M}^{\prime \prime}(2.1 .2)$}

Let us consider the 'less intimidating' sum first. We want to show that there exists a $C^{\prime \prime} \in \mathbb{R}$ such that $\left|S_{q_{n}}^{\prime \prime}\right| \leq C^{\prime \prime}$ for all $n \in \mathbb{N}$.

Note that in [3], Hardy and Litt lew o od prove a similar theorem.

Theorem 2.1 (Hardy, Littlew o od). Let $\alpha$ be badly-approximable. Then there exists $C^{*}>0$ such that $\left|S_{M}^{\prime \prime}\right| \leq C^{*}$ for each $M \in \mathbb{N}^{+}$.

We proceed by calculating real and imaginary parts.

$$
\frac{1}{e(m \alpha)-1}=-\frac{1}{2}-\frac{i}{2} \cot (\pi m \alpha) \text {. }
$$

The Taylor series expansion of $\cot x$ is

$$
\cot x=\sum_{n=0}^{\infty} \frac{(-1)^{n} 4^{n} B_{2 n}}{(2 n) !} x^{2 n-1}=\frac{1}{x}-\frac{x}{3}+\frac{x^{3}}{45}-\cdots
$$

with radius of convergence $0<|x|<\pi$. Here $B_{n}$ is the $n$th Bernoulli number. 


\section{UPPER BOUNDS FOR DOUBLE EXPONENTIAL SUMS ALONG A SUBSEQUENCE}

Note that due to the symmetry of $\cot x$,

$$
\cot (\pi m \alpha)=\cot (\pi\{\{m \alpha\}\}) .
$$

So we can write

$$
\cot (\pi m \alpha)=\frac{1}{\pi\{\{m \alpha\}\}}\left(1+\sum_{n=1}^{\infty} \frac{(-1)^{n} 4^{n} B_{2 n}}{(2 n) !}(\pi\{\{m \alpha\}\})^{2 n}\right) .
$$

Now the series on the right is negative and it takes values strictly between 0 (when $\{\{m \alpha\}\}$ is close to 0 ) and -1 (when $\{\{m \alpha\}\}$ is close to $\pm \frac{1}{2}$ ).

Hence, in order to prove that $\left|S_{q_{n}}^{\prime \prime}\right|$ is bounded by a uniform constant for all $n \in \mathbb{N}$, we have to prove the following:

Lemma 2.2. Let $\alpha \in \mathbb{R}$. Then there exists $C=C(\alpha)>0$ such that,

$$
\left|\sum_{m=1}^{q_{n}-1} \frac{1}{q_{n}\{\{m \alpha\}\}}\right| \leq C \quad \text { for all } n \in \mathbb{N} .
$$

We will consider two different proofs of Lemma 2.2. The first one is simpler, while the latter one will be applicable to estimating $S_{M}^{\prime}$ as well. The second proof is also malleable to proving Theorem 2.1

\subsection{Koksma-Hlawka Proof of Lemma 2.2}

Recall the Koksma-Hlawka inequality.

LEMма 2.3. Let $f$ be a real function with period 1 of bounded variation. Then for every sequence $\left\{x_{m}\right\}$ and every integer $N \geq 1$,

$$
\left|\frac{1}{N} \sum_{m=1}^{N} f\left(x_{m}\right)-\int_{0}^{1} f(x) \mathrm{dx}\right| \leq V(f) \frac{D_{N}\left(x_{m}\right)}{N},
$$

where $V(f)$ is the total variation of the function.

We wish to apply this inequality with

$$
f(x)=\frac{1}{\{\{x\}\}}, \quad x_{m}=\{m \alpha\}, \quad \text { and } \quad N=q_{n}-1 .
$$

Therefore we have to restrict the domain on which we define our function, in order to ensure that it is integrable.

We are able to use the following from [8]

$$
\left|\alpha-\frac{p_{n-1}}{q_{n-1}}\right|>\frac{1}{2 q_{n-1} q_{n}} .
$$




\section{CHRISTOPHER J. WHITE}

So, for all $m \leq N=q_{n}-1$, we have

$$
\|m \alpha\|>\frac{1}{2 q_{n}} .
$$

Hence we can restrict the domain of $f$ to the interval $\left[\frac{1}{2 q_{n}}, 1-\frac{1}{2 q_{n}}\right]$. Furthermore, since $f$ is anti-symmetric about $1 / 2$, the integral of $f$ over this interval is equal to 0 . The total variation, $V(f)$, of $f$ is

$$
\sup _{\mathcal{P}} \sum_{i=1}^{n_{p}}\left|\frac{1}{\left\{\left\{x_{i+1}\right\}\right\}}-\frac{1}{\left\{\left\{x_{i}\right\}\right\}}\right|,
$$

where $\mathcal{P}$ is a partition of $\left[\frac{1}{2 q_{n}}, 1-\frac{1}{2 q_{n}}\right]$. As $f$ is monotone in this interval, the total variation is maximised when we take the trivial partition (that is the two endpoints). Therefore $V(f)=4 q_{n}$.

Finally, we move on to considering the Discrepancy.

Lemma 5.6 from [5] states that

where

$$
D_{N}(m \alpha) \leq 3 \sum_{j=0}^{r} t_{j},
$$

$$
N=\sum_{j=0}^{r} q_{j} t_{j}
$$

is the Ostrowski expansion of $N$. This is defined in the next subsection (see Definition 2.5) but all we need to know here is that if $N=q_{n}$, then $t_{n}=1$ and $t_{i}=0$ for all $i \neq n$. So $D_{q_{n}}(m \alpha) \leq 3$.

Finally, we can apply all the estimates we have (with $N=q_{n}-1$ and $f \&$ $\left\{x_{m}\right\}$ as above.)

$$
\begin{aligned}
\left|\sum_{m=1}^{q_{n}-1} f\left(x_{m}\right)-\left(q_{n}-1\right) \int_{0}^{1} f(x) \mathrm{dx}\right| & \leq D_{q_{n}-1}\left(x_{m}\right) V(f) \\
& \leq\left(D_{q_{n}}\left(x_{m}\right)+1\right) V(f) \\
& \leq 4 \cdot 4 q_{n}=16 q_{n}
\end{aligned}
$$

Hence,

$$
\left|\frac{1}{q_{n}} \sum_{m=1}^{q_{n}-1} \frac{1}{\{\{m \alpha\}\}}\right| \leq 16 .
$$

Here we used the obvious fact that $D_{M}\left(x_{m}\right) \leq D_{M+1}\left(x_{m}\right)+1$. 


\subsection{The sum $S_{M}^{\prime}(2.1 .1)$}

We move on to considering the sum

$$
S_{M}^{\prime}:=\frac{1}{M} \sum_{m=1}^{M-1} \frac{e(M m \alpha)}{e(m \alpha)-1} .
$$

We will write this sum as a telescoping series and then take advantage of some cancellation to reduce our situation to considering the sum $S_{M}^{\prime \prime}(2.1 .2)$. Firstly,

$$
\begin{aligned}
\sum_{m=1}^{M-1} \frac{e(M m \alpha)}{e(m \alpha)-1}= & \sum_{m=1}^{M-1}(e(M m \alpha)-e(M(m+1) \alpha)) \sum_{k=1}^{m} \frac{1}{e(k \alpha)-1} \\
& +e\left(M^{2} \alpha\right) \sum_{k=1}^{M-1} \frac{1}{e(k \alpha)-1} .
\end{aligned}
$$

We then consider the outer part of the sum on the right hand side of (2.4.1) (for $M=q_{n}$ ),

$$
\begin{aligned}
e\left(m q_{n} \alpha\right)-e\left((m+1) q_{n} \alpha\right) & =e\left(m q_{n} \alpha\right)-e\left((m+1) q_{n} \alpha\right) \\
& =e\left(m q_{n} \alpha\right)-e\left(m q_{n} \alpha\right) e\left(q_{n} \alpha\right) \\
& =\left(1-e\left(q_{n} \alpha\right)\right) e\left(m q_{n} \alpha\right) .
\end{aligned}
$$

In absolute value this is less than $2 \pi / q_{n+1}$.

Now using the triangle inequality and Lemma 2.2, we see that (2.1.3) results from the following lemma.

Lemma 2.4. For all $\alpha \in \mathbb{R} \backslash \mathbb{Q}$ and for all $m \leq q_{n}-1$,

$$
\sum_{k=1}^{m} \frac{1}{\{\{k \alpha\}\}}=O\left(q_{n} \cdot \max _{i \leq n}\left\{1, \log a_{i}\right\}\right) .
$$

To prove this Lemma we will need to introduce some different techniques, which will also yield a new proof of Lemma 2.2.

\subsection{The Ostrowski proof of Lemmas 2.2 and 2.4}

Our alternative proof of Lemma 2.2 will involve decomposing the sum in (2.2.1) into segments where there is some obvious cancellation.

Definition 2.5. Let $\alpha$ be irrational. Then for every $n \in \mathbb{N}$ there exists a unique integer $M \geq 0$ and a unique sequence $\left\{c_{k+1}\right\}_{k=0}^{\infty}$ of integers such that

$$
q_{M} \leq m<q_{M+1} \quad \text { and } \quad m=\sum_{k=0}^{\infty} c_{k+1} q_{k},
$$




\section{CHRISTOPHER J. WHITE}

with

and

$$
\begin{aligned}
& 0 \leq c_{1}<a_{1}, 0 \leq c_{k+1} \leq a_{k+1} \quad \text { for } \quad k \geq 1 \\
& c_{k}=0 \quad \text { whenever } \quad c_{k+1}=a_{k+1} \quad \text { for some } k \geq 1
\end{aligned}
$$

$$
c_{k+1}=0 \quad \text { for } \quad k>M .
$$

This is known as the Ostrowski expansion.

We will consider segments of our sum which 'spread out' in the unit interval. We take our inspiration from a set of intervals discussed in [6].

Definition 2.6 (Special intervals). For fixed $\alpha$ define $\mathcal{A}(i)$ to be the collection of non-negative integers $n$ with Ostrowski expansions of the form

$$
n=\sum_{k=i}^{\infty} c_{k+1} q_{k} .
$$

Then for each $i \in \mathbb{N}$ and for each $\gamma \in \mathbb{R} / \mathbb{Z}$ we define a subset $\mathcal{J}(i, \gamma)$ (which turns out to be an interval, see [6]) of $\mathbb{R} / \mathbb{Z}$ by

$$
\mathcal{J}(i, \gamma)=\gamma+\overline{\{n \alpha: n \in \mathcal{A}(i)\}}
$$

These intervals have some very nice properties such as

$$
\sup _{N \in \mathbb{N}} \sup _{\mathcal{J} \subseteq \mathbb{R} / \mathbb{Z}}\left|\sum_{n=1}^{N} \chi_{\mathcal{J}}(n \alpha)-N \cdot\right| \mathcal{J}|| \leq K,
$$

where $K$ is a universal constant and the inner supremum is taken over all special intervals $\mathcal{J}$ for $\alpha$. We will use what these intervals tell us about the distribution of $n \alpha$ on the unit interval to achieve cancellation in (2.2.1).

Let

and

$$
m=\sum_{i=0}^{n-1} c_{i+1} q_{i} \leq q_{n}-1, \quad 0 \leq c_{i+1} \leq a_{i+1}
$$

$$
n(i, c):=\sum_{j=0}^{i-1} c_{j+1} q_{j}+c q_{i} .
$$

We will use this decomposition to sum up to $m$.

$$
\sum_{k=1}^{m} \frac{1}{\{\{k \alpha\}\}}=\sum_{i=0}^{n-1} \sum_{c=0}^{c_{i+1}-1} \sum_{l=n(i, c)+1}^{n(i, c)+q_{i}} \frac{1}{\{\{l \alpha\}\}} .
$$

Note that

$$
n(i, c)+q_{i}=n(i, c+1) \quad \text { and } \quad n\left(i, c_{i+1}-1\right)+q_{i}=n(i+1,0) .
$$




\section{UPPER BOUNDS FOR DOUBLE EXPONENTIAL SUMS ALONG A SUBSEQUENCE}

Let us consider a situation, where we are studying

$$
\sum_{l=n(i, c)+1}^{n(i, c)+q_{i}} \frac{1}{\{\{l \alpha\}\}}
$$

We wish to approximate $\alpha$ by $p_{i} / q_{i}$ and achieve (almost) complete cancellation in the main term that we get.

Obviously, problems can occur. Specifically, if $l \cdot p_{i} \equiv 0\left(q_{i}\right)$, then we do not want to divide by 0 , so we want to isolate these terms and deal with them separately. Note that since $\left(p_{i}, q_{i}\right)=1$, we have a complete set of residue classes modulo $q_{i}$, so in each sum (2.5.1) we will have exactly one term, $l=(c+1) q_{i}$, where this happens. Also, there exists $r \leq q_{i}$ such that

$$
\begin{gathered}
n(i, 0)+r=q_{i}, \\
n(i, 1)+r=2 q_{i}, \\
\vdots \\
n\left(i, c_{i+1}-1\right)+r=c_{i+1} q_{i} .
\end{gathered}
$$

So we can consider all of these terms separately.

Finally, we consider summing over a complete set of residue classes modulo $q_{i}$. We will first consider the simple case, $\left(1 \leq k \leq q_{i-1}\right)$, which will give us a second proof of Lemma 2.2. We write

Now

$$
\alpha=\frac{p_{i}}{q_{i}}+\frac{\xi_{i}}{q_{i} q_{i+1}}, \quad \text { where } \quad \frac{1}{2}<\left|\xi_{i}\right|<1 .
$$

$$
\sum_{k=1}^{q_{i}-1} \frac{1}{\{\{k \alpha\}\}}=\sum_{k=1}^{q_{i}-1} \frac{1}{\left\{\left\{k \frac{p_{i}}{q_{i}}+\frac{k \xi_{i}}{q_{i} q_{i+1}}\right\}\right\}} .
$$

Now we use the fact that

$$
\left\{\left\{\frac{k p_{i}}{q_{i}}+\frac{k \xi_{i}}{q_{i} q_{i+1}}\right\}\right\}=\left\{\left\{\frac{k p_{i}}{q_{i}}\right\}\right\}+\left\{\left\{\frac{k \xi_{i}}{q_{i} q_{i+1}}\right\}\right\},
$$

unless perhaps if $k p_{i} \equiv \frac{q_{i}}{2}$ modulo $q_{i}$ (when $2 \mid q_{i}$ ), or if $k p_{i} \equiv \frac{q_{i} \pm 1}{2}$ (when $2 \mid q_{i}+1$ ). Now (2.5.2) equals 1

$$
\sum_{k=1}^{q_{i-1}} \frac{1}{\left\{\left\{k \frac{p_{i}}{q_{i}}\right\}\right\}}\left(\frac{1}{1+\left\{\left\{\frac{k p_{i}}{q_{i}}\right\}\right\}^{-1} \frac{k \xi_{i}}{q_{i} q_{i+1}}}\right)+O(1) .
$$

\footnotetext{
${ }^{1}$ The one or two extra term/s mentioned just above have been removed from the sum and are accounted for by the $O(1)$ term.
} 
Furthermore,

$$
\begin{aligned}
\left(\frac{1}{1+\left\{\left\{\frac{k p_{i}}{q_{i}}\right\}\right\}^{-1} \frac{k \xi_{i}}{q_{i} q_{i+1}}}\right)=1 & -\left\{\left\{\frac{k p_{i}}{q_{i}}\right\}\right\}^{-1} \frac{k \xi_{i}}{q_{i} q_{i+1}} \\
& +\left\{\left\{\frac{k p_{i}}{q_{i}}\right\}\right\}^{-2}\left(\frac{k \xi_{i}}{q_{i} q_{i+1}}\right)^{2}-\cdots
\end{aligned}
$$

There exists $n_{k}$ such that $1 \leq n_{k} \leq q_{i}-1$ and $n_{k} \equiv k p_{i} \bmod q_{i}$. Now we define $n_{k}^{\prime}$ as follows

$$
n_{k}^{\prime}:= \begin{cases}n_{k}, & n_{k} \leq \frac{q_{i}}{2} \\ n_{k}-q_{i}, & n_{k}>\frac{q_{i}}{2}\end{cases}
$$

Then,

$$
\left\{\left\{\frac{k p_{i}}{q_{i}}\right\}\right\}^{-1} \frac{k \xi_{i}}{q_{i} q_{i+1}}=\frac{k \xi_{i}}{n_{k}^{\prime} q_{i+1}} .
$$

We then know that for all $k$,

$$
\left\{\left\{\frac{k p_{i}}{q_{i}}\right\}\right\}^{-1} \frac{k \xi_{i}}{q_{i} q_{i+1}}+\left\{\left\{\frac{k p_{i}}{q_{i}}\right\}\right\}^{-2}\left(\frac{k \xi_{i}}{q_{i} q_{i+1}}\right)^{2}-\cdots=C_{k} \frac{k \xi_{i}}{n_{k}^{\prime} q_{i+1}} .
$$

We need $\left|n_{k}^{\prime}\right| \geq 2$ in order to have a uniform bound over $k$ for the constant $C_{k}$. When this is the case

$$
-2<C_{k}<-\frac{1}{2}
$$

(apart from the one or two exceptions mentioned previously.) So we have to isolate another two terms. We write $k_{1}, k_{-1}$ for the numbers, where $k_{1} p_{i} \equiv 1$ $\bmod q_{i}$ and $k_{-1} p_{i} \equiv-1 \bmod q_{i}$, respectively. So (2.5.2) becomes

$$
\begin{array}{r}
\sum_{n_{k}=2}^{q_{i}-2}\left(\frac{1}{\left\{\left\{\frac{n_{k}}{q_{l}}\right\}\right\}}+C_{k}\left(\frac{k \xi_{i} q_{i}}{\left(n_{k}^{\prime}\right)^{2} q_{i+1}}\right)\right)+\frac{1}{\left\{\left\{k_{1} \alpha\right\}\right\}}+\frac{1}{\left\{\left\{k_{-1} \alpha\right\}\right\}}+O(1) \\
=\sum_{n_{k}=2}^{q_{i}-2} C_{k}\left(\frac{k \xi_{i} q_{i}}{\left(n_{k}^{\prime}\right)^{2} q_{i+1}}\right)+O\left(q_{i}\right) .
\end{array}
$$

(Here we used the basic approximation from $\mathrm{Kh}$ in $\mathrm{chin}$ (2.3.1) to deal with the two extra terms.) 


\section{UPPER BOUNDS FOR DOUBLE EXPONENTIAL SUMS ALONG A SUBSEQUENCE}

By the rearrangement inequality, (see 4], Theorem 368), this first sum is smaller (in modulus) than

$$
4 q_{i}\left(\frac{1}{2^{2}}+\frac{1}{3^{2}}+\cdots\right)
$$

which in turn is bounded above by $4 q_{i}$. So

$$
\sum_{k=1}^{q_{i}-1} \frac{1}{\{\{k \alpha\}\}}=O\left(q_{i}\right)
$$

as required. Now, we move on to a proof of Lemma 2.4. We wish to prove, (for all $i$ ), that

$$
\sum_{c=0}^{c_{i+1}-1} \sum_{l=n(i, c)+1}^{n(i, c)+q_{i}} \frac{1}{\{\{l \alpha\}\}}=O\left(q_{i+1} \log c_{i+1}\right) .
$$

Note that if we sum

$$
\sum_{l=n(i, c)+1}^{n(i, c)+q_{i}} \frac{1}{\{\{l \alpha\}\}}
$$

then a similar argument to the proof of Lemma 2.2 shows that this is equal to

$$
O\left(q_{i}\right)+\frac{1}{\left\{\left\{k_{(1, c)} \alpha\right\}\right\}}+\frac{1}{\left\{\left\{k_{(-1, c)} \alpha\right\}\right\}}+\frac{1}{\left\{\left\{(c+1) q_{i} \alpha\right\}\right\}},
$$

where

$$
n(i, c)+1 \leq k_{( \pm 1, c)} \leq n(i, c)+q_{i}, \quad \text { and } \quad k_{( \pm 1, c)} p_{i} \equiv \pm 1 \quad \bmod q_{i} .
$$

Clearly,

$$
k_{( \pm 1, c)}=k_{( \pm 1,0)}+c q_{i}
$$

Furthermore, as $n(i, 0)<q_{i}$,

$$
k_{\left( \pm 1, c_{i+1}-r\right)}<n\left(i,\left(c_{i+1}-r\right)\right)+q_{i}<q_{i+1}-(r-1) q_{i} .
$$

Now, we calculated earlier that

$$
\frac{1}{\{\{k \alpha\}\}}=\frac{1}{\left\{\left\{k \frac{p_{i}}{q_{i}}\right\}\right\}}\left(\frac{1}{1+\left\{\left\{\frac{k p_{i}}{q_{i}}\right\}\right\}^{-1} \frac{k \xi_{i}}{q_{i} q_{i+1}}}\right) .
$$

Letting $k=k_{(1,0)}$,

$$
\begin{aligned}
\frac{1}{\left\{\left\{k_{(1,0)} \alpha\right\}\right\}} & =q_{i}\left(\frac{1}{1+\frac{k_{(1,0)} \xi_{i}}{q_{i+1}}}\right) \\
& =\frac{q_{i} q_{i+1}}{q_{i+1}+k_{(1,0)} \xi_{i}} .
\end{aligned}
$$


Hence,

$$
\frac{1}{\left\{\left\{k_{(1, c)} \alpha\right\}\right\}}=\frac{q_{i} q_{i+1}}{q_{i+1}+\left(k_{(1,0)}+c q_{i}\right) \xi_{i}}
$$

and also

$$
\frac{1}{\left\{\left\{k_{(-1, c)} \alpha\right\}\right\}}=\frac{-q_{i} q_{i+1}}{q_{i+1}-\left(k_{(-1,0)}+c q_{i}\right) \xi_{i}} .
$$

Now, without loss of generality, assume that $\xi_{i}>0$. Then

$$
\frac{1}{\left\{\left\{k_{(1, c)} \alpha\right\}\right\}}<q_{i} \text { for all } c .
$$

Hence,

$$
\begin{aligned}
\sum_{c=0}^{c_{i+1}-1} \sum_{l=n(i, c)+1}^{n(i, c)+q_{i}} \frac{1}{\{\{l \alpha\}\}}= & O\left(q_{i+1}\right)+\sum_{c=0}^{c_{i+1}-1} \frac{1}{\left\{\left\{(c+1) q_{i} \alpha\right\}\right\}} \\
& +\sum_{c=0}^{c_{i+1}-1} \frac{-q_{i} q_{i+1}}{q_{i+1}-\left(k_{(-1,0)}+c q_{i}\right) \xi_{i}} \\
= & O\left(q_{i+1}\right)+O\left(q_{i+1} \log c_{i+1}\right) \\
& +\sum_{c=0}^{c_{i+1}-1} \frac{-q_{i} q_{i+1}}{q_{i+1}-\left(k_{(-1,0)}+c q_{i}\right) \xi_{i}}
\end{aligned}
$$

Finally, (using $k_{(-1,0)} \leq q_{i}+q_{i-1}$ and $\xi<1$ ),

$$
\begin{aligned}
\left|\sum_{c=0}^{c_{i+1}-1} \frac{-q_{i} q_{i+1}}{q_{i+1}-\left(k_{(-1,0)}+c q_{i}\right) \xi_{i}}\right| & \leq \frac{q_{i+1}}{c_{i+1}-1}+\cdots+\frac{q_{i+1}}{2}+q_{i+1}+2 q_{i+1} \\
& =O\left(q_{i+1} \log c_{i+1}\right) .
\end{aligned}
$$

As this is true for all $i$, the condition for Lemma 2.4 follows.

REMARK 2.7. Equation (1.13) in [1] tells us that the sum

$$
\sum_{c=0}^{c_{i+1}-1} \frac{1}{\left\{\left\{(c+1) q_{i} \alpha\right\}\right\}}
$$

can be no smaller than $O\left(q_{i+1} \log c_{i+1}\right)$. 


\section{UPPER BOUNDS FOR DOUBLE EXPONENTIAL SUMS ALONG A SUBSEQUENCE}

REMARK 2.8. In our final calculation we have ignored the cancellation between the positive and negative terms. However, when $c_{i+1} \approx a_{i+1} / 2$, for example, we get very little cancellation and our main term is

$$
O\left(q_{i+1} \log a_{i+1}\right) .
$$

REMARK 2.9. In fact since convergents $p_{i} / q_{i}$ give lower bounds for $\alpha$ when $i$ is even this implies that $\xi_{i}$ is positive when $i$ is even (and negative when $i$ is odd). Now from the well-known formula for continued fractions

$$
p_{i-1} q_{i}-p_{i} q_{i-1}=(-1)^{i},
$$

we see that for the negative terms we separated,

$$
k_{(-1, c)}=c q_{i}+q_{i-1} .
$$

These correspond to the semiconvergents

$$
\frac{c p_{i}+p_{i-1}}{c q_{i}+q_{i-1}}
$$

in the continued fraction expansion of $\alpha$.

ACKnowledgment. The author would like to thank A lan $\mathrm{H}$ a y n es for his invaluable advice and tutelage, and $\mathrm{Jens} \mathrm{Marklof}$ for pointing out the work of Cosentino and Flaminio.

\section{REFERENCES}

[1] BERESNEVICH, V.-HAYNES, A.-VELANI, S.: Sums of reciprocals of $($ n $\alpha-\gamma)$ modulo 1 and multiplicative Diophantine approximation, preprint.

[2] COSENTINO, S.-FLAMINIO, L.: Equidistribution for higher-rank abelian actions on Heisenberg nilmanifolds, J. Mod. Dyn. 9 (2015), 305-353.

[3] HARDY, G. H.-LITTLEWOOD, J. E.: Some problems of Diophantine approximation (a series of cosecants), Bull. Calcutta Math. Soc. 20 (1930), 251-266.

[4] HARDY, G. H-LitTlEWOOD, J. E.-PÓLYA, G.: Inequalities, Cambridge Mathematical Library (2nd edition), Cambridge University Press, Cambridge, 1952.

[5] HARMAN, G.: Metric Number Theory, In: London Mathematical Society Monographs. New Series, Vol. 18. The Clarendon Press, Oxford University Press, New York, 1998.

[6] HAYNES, A.: Equivalence classes of codimension one cut-and-project sets, Ergodic Theory Dyn. Syst. 36 (2016), no. 3, 816-831.

[7] KHINCHIN, A. YA.: Continued Fractions, The University of Chicago Press, Chicago, III.-London, 1964. 


\section{CHRISTOPHER J. WHITE}

[8] ROCKETT, A.-SZÜSZ, P.: Continued Fractions, World Scientific Publishing Co., Inc., River Edge, NJ, 1992.

[9] SINAI, YA. G.-ULCGRAI, C.: Estimates from above of certain double trigonometric sums, J. Fixed Point Theory Appl. 6 (2009), no. 1, 93-113.

Received November 1, 2015

Accepted October 10, 2016

\section{Christopher J. White}

School of Mathematics

University of Bristol

Bristol

U.K.

E-mail: chris.white@bristol.ac.uk 